Louisiana State University

LSU Digital Commons

$5-4-2015$

\title{
Direct measurement of the quantum density matrix in the basis of azimuthal angle
}

\author{
Mohammad Mirhosseini \\ University of Rochester Institute of Optics \\ Omar S. Magaña-Loaiza \\ University of Rochester Institute of Optics \\ Seyed Mohammad Hashemi Rafsanjani \\ University of Rochester Institute of Optics \\ Changchen Chen \\ University of Rochester Institute of Optics \\ Ebrahim Karimi \\ University of Ottawa
}

See next page for additional authors

Follow this and additional works at: https://digitalcommons.Isu.edu/physics_astronomy_pubs

\section{Recommended Citation}

Mirhosseini, M., Magaña-Loaiza, O., Rafsanjani, S., Chen, C., Karimi, E., \& Boyd, R. (2015). Direct measurement of the quantum density matrix in the basis of azimuthal angle. CLEO: QELS - Fundamental Science, CLEO_QELS 2015, 1551p. https://doi.org/10.1364/CLEO_QELS.2015.FTu4A.2

This Conference Proceeding is brought to you for free and open access by the Department of Physics \& Astronomy at LSU Digital Commons. It has been accepted for inclusion in Faculty Publications by an authorized administrator of LSU Digital Commons. For more information, please contact ir@lsu.edu. 


\section{Authors}

Mohammad Mirhosseini, Omar S. Magaña-Loaiza, Seyed Mohammad Hashemi Rafsanjani, Changchen Chen, Ebrahim Karimi, and Robert W. Boyd 


\title{
Direct measurement of the quantum density matrix in the basis of azimuthal angle
}

\author{
Mohammad Mirhosseini ${ }^{1, *}$, Omar S. Magaña-Loaiza ${ }^{1}$, \\ Seyed Mohammad Hashemi Rafsanjani ${ }^{1}$, Changchen Chen ${ }^{1}$, \\ Ebrahim Karimi ${ }^{2}$, and Robert W. Boyd ${ }^{1,2}$ \\ ${ }^{1}$ The Institute of Optics, University of Rochester, Rochester, New York 14627, USA \\ ${ }^{2}$ Department of Physics, University of Ottawa, Ottawa ON K1N 6N5, Canada \\ *mirhosse@optics.rochester.edu
}

\begin{abstract}
We theoretically propose and experimentally demonstrate a method for directly measuring the density matrix of an unknown quantum system in the basis of azimuthal angle. We apply our method for characterizing 7-dimensional pure and mixed superpositions of orbital-angular-momentum modes.
\end{abstract}

(C) 2014 Optical Society of America

OCIS codes: $270.0270,260.5585$.

The structure of optical field has been long a subject of study in a variety of topics in physical optics such as diffraction, imaging, and coherence theories [1]. More recently, the transverse state of a single photon has been identified as a valuable resource for encoding information [2,3], and as an additional degree of multiplexing for increasing the channel capacity of classical communication [4].

The "wavefunction" of a photon can be directly measured using weak values [5]. Although this measurement scheme can be realized efficiently for pure states [6], the characterization of mixed quantum states remains a challenging task [7]. Here, we demonstrate a photon-efficient method for directly measuring the quantum density matrix in the angular representation. Unlike the previous schemes based on weak-values, our experiment utilizes a sequence of strong measurements to directly find the real and the imaginary parts of the density matrix elements, without any requirement for post-processing. Further, we show that the inherent relation between the azimuthal angle and the orbital angular momentum (OAM) can be used to characterize pure and mixed OAM states.

We describe the quantum state of interest with the (unknown) density matrix $\hat{\rho}$. The pure state representation of $\hat{\rho}$ reads as $\hat{\rho}=\sum_{i} p_{i}\left|\psi_{i}\right\rangle\left\langle\psi_{i}\right|$. In order to measure $\hat{\rho}$ in the spatial representation, we utilize the polarization degree of freedom as a pointer. We initially prepare the photons in the diagonal polarization state

$$
\hat{\chi}=\hat{\rho} \otimes|D\rangle\langle D|
$$

where, $|D\rangle=(|H\rangle+|V\rangle) / \sqrt{2}$. We now consider a polarization sensitive rotation of the state, realized via a coupling between the spin and OAM variables

$$
\hat{U} \hat{\chi} \hat{U}^{\dagger}=\sum_{i} p_{i}\left(\hat{T}\left|\psi_{i}\right\rangle \otimes|H\rangle+\hat{T}^{\dagger}\left|\psi_{i}\right\rangle \otimes|V\rangle\right)\left(\left\langle\psi_{i}\right| \otimes\langle H| \hat{T}^{\dagger}+\left\langle\psi_{i}\right| \otimes\langle V| \hat{T}\right) .
$$

Here, $\hat{U}=e^{i \tau \hat{L}_{z} \otimes \hat{\sigma}_{z}}$ is the measurement's evolution operator, $\hat{T}=e^{i \tau \hat{L}_{z}}$ is the rotation operator, and $\tau$ is the angle of rotation. We have previously studied the act of the measurement operator defined above for the special case of weak interactions [8]. Here, however, we are considering the rotation angle to be an arbitrary small or large angle $\tau \in[0,2 \pi]$. Consider the post-selection of the evolved state on an eigenstates of angle $|\theta\rangle$

$$
\left\langle\theta\left|\hat{U} \hat{\chi} \hat{U}^{\dagger}\right| \theta\right\rangle=\sum_{i} p_{i}\left(\left\langle\theta_{-} \mid \psi_{i}\right\rangle|H\rangle+\left\langle\theta_{+} \mid \psi_{i}\right\rangle|V\rangle\right)\left(\left\langle\psi_{i} \mid \theta_{-}\right\rangle\langle H|+\left\langle\psi_{i} \mid \theta_{+}\right\rangle\langle V|\right),
$$

where $\theta_{-}=[\theta-\tau]$ and $\theta_{+}=[\theta+\tau]$. After the post-selection, the spatial state of the photon collapses to $|\theta\rangle$ and all the information about the density matrix $\hat{\rho}$ is transferred to the polarization state of the pointer. Since the post-selection is a non-unitary operation, we need to normalize the resulting polarization state (of the pointer) by dividing it by $N(\theta, \tau)=\sum_{i} p_{i}\left(\left|\left\langle\psi_{i} \mid \theta_{-}\right\rangle\right|^{2}+\left|\left\langle\psi_{i} \mid \theta_{+}\right\rangle\right|^{2}\right)=\left\langle\theta_{-}|\hat{\rho}| \theta_{-}\right\rangle+\left\langle\theta_{+}|\hat{\rho}| \theta_{+}\right\rangle$. The real and the imaginary parts of the density matrix can then be simply found by characterizing the polarization state of the pointer as

$$
\left\langle\theta_{+}|\hat{\rho}| \theta_{-}\right\rangle=\frac{N(\theta, \tau)}{2}\left[\left\langle\hat{\sigma}_{x}\right\rangle+i\left\langle\hat{\sigma}_{y}\right\rangle\right] .
$$



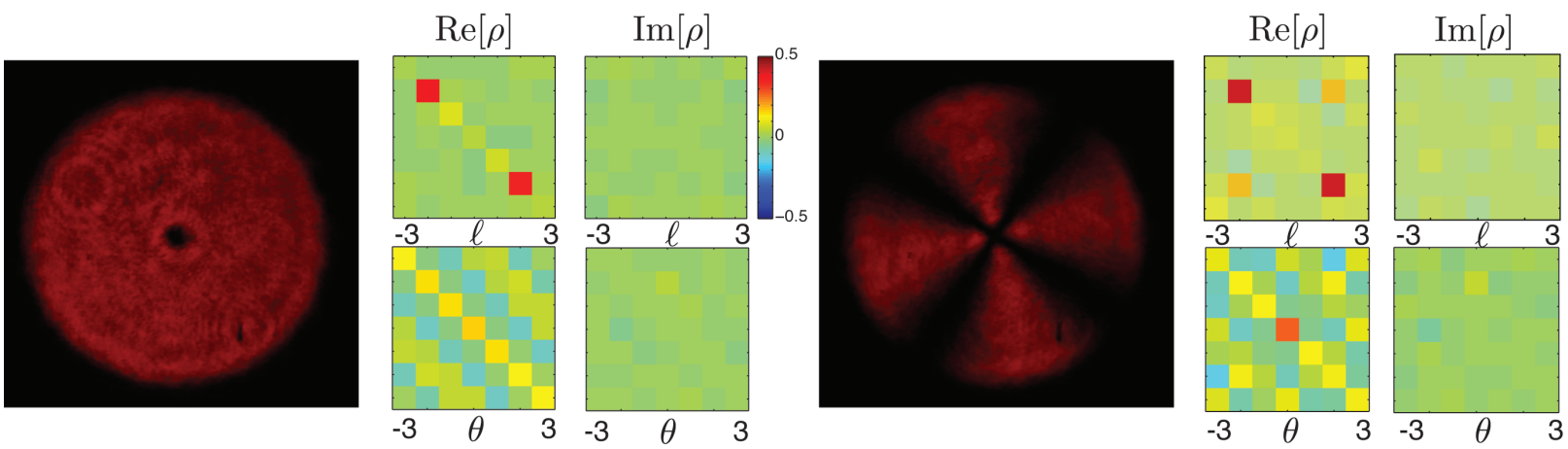

Fig. 1. Experimental data for characterizing a mixed superposition of two OAM modes is displayed on the left. The measurement results for a pure superposition of the same modes is shown to the right.

Here, $\hat{\sigma}_{x}$ and $\hat{\sigma}_{y}$ are the Pauli matrices and their expectation values can be found by measuring the Stokes parameters in the diagonal and circular polarization bases, respectively.

We have realized the measurement operator $\hat{U}=e^{i \tau \hat{L}_{z} \otimes \hat{\sigma}_{z}}$ by using a Sagnac interferometer. The input beam is separated to two polarization components that propagate inside the interferometer in the opposite directions. A Dove prism placed inside the interferometer rotates the field distribution of two polarization components towards different directions by the angle $\tau$. The value of rotation angle can be set to any arbitrary value by setting the axis of the Dove prism. The two polarization components are combined with no loss in the output port of the polarizing beam splitter. We simultaneously perform the post-selection on all possible angular states $|\theta\rangle$ by taking a picture of the output beam with a charge-coupled device (CCD) camera. A polarizer is placed before the camera for project the polarization state of the output beam either in the diagonal/anti-diagonal or the right-handed/left-handed bases.

We have experimentally characterized pure and mixed superpositions of OAM modes in the angular basis. The pure states are generated using a binary grating realized on a digital micro-mirror device (DMD) [9]. A mixed state is created by rapidly switching between two or more pure states. Figure, 1 shows the intensity structure of two states from the experiment. We discretize the Hilbert state of azimuthal angle to 7 segments. The density matrix in the angular representation is directly found from the experiment as shown in the analysis above. Once we have measured the density matrix in the angular basis, we have used the Fourier relation to find the density matrix in the OAM representation. It can be observed that the existence of the coherence terms in the density matrix give rise to the petals in the intensity pattern of a pure state (Fig., 1, right), whereas the lack of these terms in a mixed state results in an incoherent sum of the intensities of the OAM components that have non-zero population. This method can be conveniently scaled for measuring quantum states of very large dimension in the angular representation.

1. B. Wolf and M. Born, "Principles of optics: electromagnetic theory of propagation, interference and diffraction of light," (1970).

2. M. Mirhosseini, O. S. Magaña-Loaiza, M. N. O’Sullivan, B. Rodenburg, M. Malik, M. Lavery, M. J. Padgett, D. J. Gauthier, and R. W. Boyd, "High-dimensional quantum cryptography with twisted light," arXiv.org (2014).

3. E. Nagali, L. Sansoni, F. Sciarrino, F. De Martini, L. Marrucci, B. Piccirillo, E. Karimi, and E. Santamato, "Optimal quantum cloning of orbital angular momentum photon qubits through Hong-Ou-Mandel coalescence," Nature Photonics 3, 720-723 (2009).

4. J. Wang, J.-Y. Yang, I. M. Fazal, N. Ahmed, Y. Yan, H. Huang, Y. Ren, Y. Yue, S. Dolinar, M. Tur, and A. E. Willner, "Terabit free-space data transmission employing orbital angular momentum multiplexing," Nature Photonics 6, 488-496 (2012).

5. J. S. Lundeen, B. Sutherland, A. Patel, C. Stewart, and C. Bamber, "Direct measurement of the quantum wavefunction." Nature 474, 188-191 (2011).

6. M. Mirhosseini, O. S. Magaña-Loaiza, S. M. Hashemi Rafsanjani, and R. W. Boyd, "Compressive Direct Measurement of the Quantum Wave Function," Physical Review Letters 113, 090,402 (2014).

7. J. Lundeen and C. Bamber, "Procedure for Direct Measurement of General Quantum States Using Weak Measurement," Physical Review Letters 108 (2012).

8. O. S. Magaña-Loaiza, M. Mirhosseini, B. Rodenburg, and R. W. Boyd, "Amplification of Angular Rotations Using Weak Measurements," Physical Review Letters 112, 200,401 (2014).

9. M. Mirhosseini, O. S. Magaña-Loaiza, C. Chen, B. Rodenburg, M. Malik, and R. W. Boyd, "Rapid generation of light beams carrying orbital angular momentum," Optics Express 21, 30,196-30,203 (2013). 